\title{
DYNAMIC SYSTEM APPROACH IN PSYCHOLOGY: PROPOSITION AND APPLICATION IN THE STUDY OF EMOTION, APPRAISAL AND COGNITIVE ACHIEVEMENT
}

\author{
Cristiano M. A. Gomes, Hudson F. Golino, Bianca C. G. Costa \\ Federal University of Minas Gerais, Belo Horizonte, Brazil \\ E-mail: cristianogomes@ufmg.br, hfgolino@gmail.com, biacgc@gmail.com
}

\begin{abstract}
Psychological processes are difficult to be studied due to their complexity. The dynamic system approach shows itself as a good tool for psychology to deal with this complexity issue. We propose two fundamental contributions of the dynamic system approach to psychology and apply it in the study of achievement emotions, appraisal and cognitive achievement. Two hypotheses were investigated: 1) More than one correlation pattern between test achievement, appraisal and emotion will be found; 2) Test achievement, appraisal and emotion form a dynamic system which will be explained by a latent variable that is dependent on the previous state of the system. A sample of thirteen students from seventh to ninth grades performed an inductive reasoning test, appraised their achievement, and declared their emotional valences (from extreme positive to extreme negative). Each variable was measured in 20 different occasions. One correlation matrix of each individual was generated and seven qualitative profiles were identified. Then four different states of relations between the variables were identified through a hidden Markov model. The two hypotheses were not refuted. It's concluded that the dynamic system approach brings new possibilities to the study of psychological processes.
\end{abstract}

Key words: achievement emotion, appraisal, cognitive achievement, dynamic system approach, methodology.

\section{Introduction}

Understanding the dynamic of cognitive processes in psychology is one of the goals of the psychological science. However, the processes studied are too complex: they have a considerable quantity of components in a net of reciprocal causality (Schmittmann et al., 2013). Despite the difficulty, there are new possibilities. We propose that the dynamic system approach can help psychology in this endeavour through two fundamental contributions. First, it defines complexity, which is a fundamental step. Accordingly to the dynamic system approach, one system is complex when: (1) the number of components interacts to define its own structure; (2) the relationship of the components is non-linear, (3) and is self-organized (Van Geert \& Steenbeek, 2008).

Learning any school content is a good example of the definition about complexity. Any learning always exceeds the input of teachers, colleagues, didactic materials, and so on, because the system has components that interact translating and transforming the content structure in function of the mechanisms of the own system (rule one). Moreover, the reciprocal influence of the components causes a non-linear dynamic (rule two) and the system progressively increases the level of the learning in a self-organized process (rule three). All these characteristics can be seen in the work of 
Mitra, Tooley, Inamdar and Dixon (2003), where, even though not relying in the dynamic systems methodologies, they show a group of children from India that improved their English pronunciation by their own using a speech-to-text software engine. Without any human mediation, their improvement is a typical example of a self-organized process.

Diminishing the complexity without losing the fundamental properties of the system is the second fundamental contribution of the dynamic system approach to psychology. According to Van Geert and Steenbeek (2005a) any study in psychology complies with the dynamic systems approach if the equation 1 is directly or indirectly present, since this is the primary equation of any dynamic system.

$$
\text { Equation 1: } y_{t+1}=f\left(y_{t}\right)
$$

The equation demonstrates that the state of the system is caused by its previous state. In other words, the value of $y$ on a specific moment of time $(t+1)$ is a function of its value on the previous moment $(\mathrm{t})$. This equation contains the fundamental idea of a dynamical system and can be applied to the study of several constructs, not being confined to a specific psychological theory (Van Geert \& Steenbeek, 2005a, p. 411). The main advantage is that despite the reduction of the complexity of the variable under investigation, it does not lead to any lost in its key properties when applying any model related to equation 1 . Accordingly to the authors, there are a variety of models in consonance with this basic equation, as the Hidden Markov Model, simulation models, differential equation, and others. These statistical and mathematical methods can reduce the complexity without losing the fundamental properties of the complex system because they maintain the basic equation 1 .

On the context of the developmental psychology there are several studies that connect the dynamic system approach to cognitive development. For example, Van Geert and Fischer (2009) and Van Geert and Steenbeek (2005b) employed dynamic systems to study the role of context-person interactions in the expression of cognitive abilities. Yan and Fischer (2002), by their turn, investigated the levels of cognitive abilities people showed in multiple contexts and scales of time. The view of humans and their psychological processes as dynamical systems is based on the idea that different components influence each other in a bidirectional (or reciprocal) way, creating a feedback loop that, in return, shapes their relation (Cramer et al., 2012a). This idea is worth not just in developmental psychology, but also in the personality research field. Cramer et al. (2012a), for example, showed evidences that personality traits emerged from the interactions between its components (thoughts, feelings and behaviors), just like flocking emerged from the local interactions between birds. The dynamic analysis presented by Cramer et al. (2012a) creates a whole new perspective of personality, since direct relations between indicators (e.g. personality items) are not just an expected characteristic, but the phenomenon under investigation.

Concluding, we proposed two fundamental contributions of the dynamic system approach to psychology that structured their relation. The first is the capacity of the dynamic system approach to objectively define complexity and its properties. The second is the capacity to reduce the complexity of the object under investigation without losing its fundamental properties. Next, we will demonstrate the relation between dynamic system and psychology theory through the study of achievement emotions.

\section{Test Achievement, Appraisal and Emotional Valence: a Dynamic System}

In the Control-Value theory of Reinhard Pekrun, achievement emotions are emotions associated with activities involving performance or outcomes (Pekrun \& Sthepens, 2010). The Control-Value theory defines emotion as the relationship between affective, cognitive, motivational, expressive and physiological components, and seeks to understand its role in academic contexts (Pekrun, 2006). The author points out two main components of achievement emotions, control and value, both tight to people's evaluation about their own achievements or outcomes (Pekrun, 2006). The first refers to the individual's judgment about the control that he has over his performance in the activity, taking into account the influence of context, other people and himself. In other words, control refers to one's judgment about how their actions affect their outcome. The second component addresses people's judgment on the importance of the activity and the outcome. 
Given control and value judgments, achievement emotions can be generated in three key moments: before, during and after performing a task (Pekrun 2006; Pekrun \& Sthepens, 2010). The theory holds that each of these moments creates different types of emotions. The first moment generates prospective emotions, linked to a previous judgment of future performance in the task itself, whereas the second moment produces activity related emotions, and the third moment generates retrospective emotions, linked to the performance achieved in the task.

The combination of control, value and the three judgment moments produces nine achievement emotions (Pekrun \& Stephens, 2010): 1) Prospective emotions: hope, relief, anxiety and despair; 2) Activity emotions: satisfaction and boredom; 3 ) Retrospective emotions: pride, shame and anger. Such emotions generated before, during and after a task have a key role in mediating the performance of a person. They will be of fundamental importance for the definition of the attitude and behavior of the person in relation to the task, thereby regulating their own performance (Pekrun, 1992; Pekrun, 2006). Thus, one's judgment on a task causes the achievement emotions, which affects their performance on the task (Pekrun, 1992). At the same time, the performance achieved in a given task will generate new emotions that will influence the judgment on future tasks. Therefore, we infer that judgment (appraisal), emotions and performance influence each other in a reciprocal and non-linear way, generating a self-organization process, characterizing a dynamic system.

\section{Research Focus}

Considering the Control-Value theory, we assume that tests composed by cycles with levels of increasing difficulty will produce a dynamic system behavior between emotion, achievement and appraisal. To investigate our assumption we'll use a testing design that incorporates four cycles. Each cycle is composed by five levels with increasing difficulty. When a cycle finishes, the next one begins with the easiest level. This process repeats until the end of the fourth cycle. Figure 1 shows this testing design. First, the person answers three items with difficulty level one (1-3 on the x-axis), then he/ she answers three items with difficulty level two (4-6 on the x-axis). The process goes on until the person answers the three most difficult items (level five). In this moment one cycle ends and the next cycle begins. People will be asked, after each group of three items, to judge their performance, i.e. if they passed or not the group of items (appraisal), and to report their perceived emotional reaction to them (levels of retrospective emotion, from extreme negative to extreme positive).

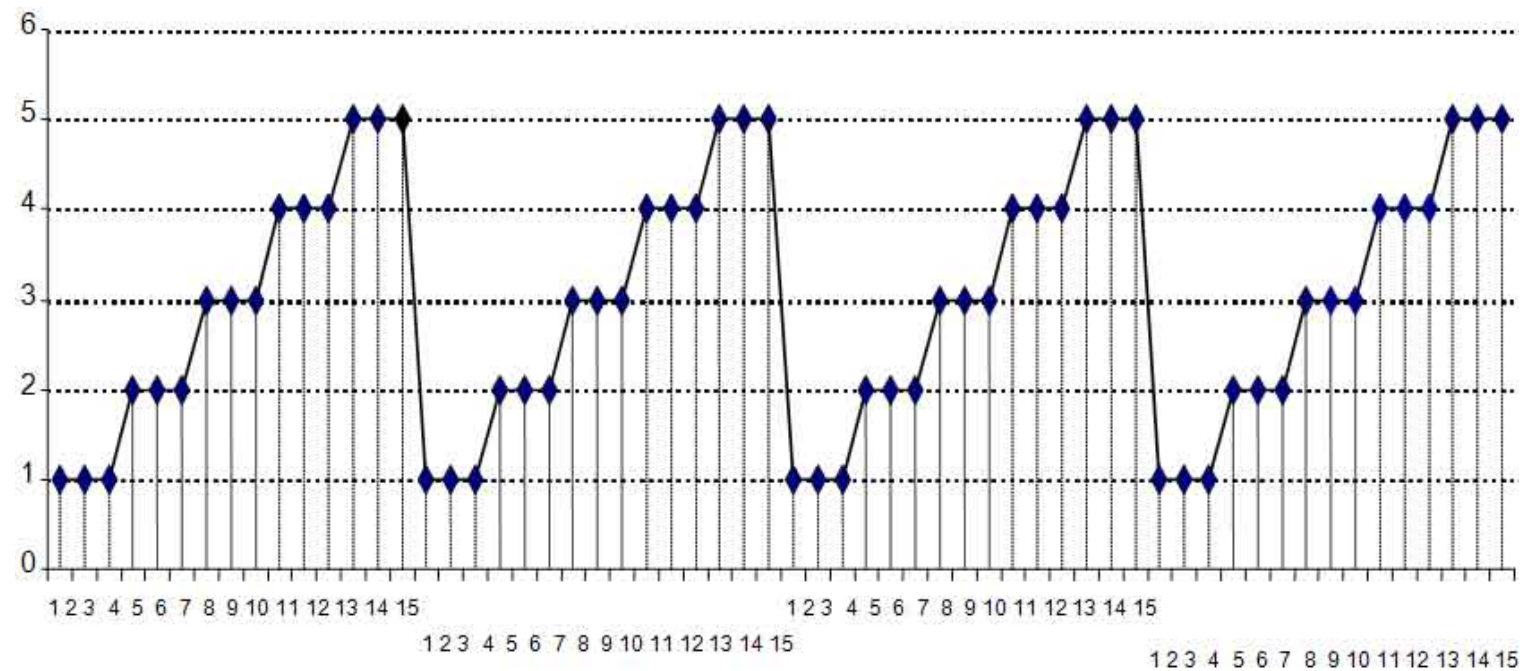

Figure 1: $\quad$ Testing Design: Difficulty Level (Y-axis) by Items (X-axis).

Two hypotheses will be investigated: 1) More than one correlation pattern between test achievement, appraisal and emotion will be found; 2) Test achievement, appraisal and emotion form a dynamic system which is explained by a latent variable $\theta$ that is dependent on the previous state of the system (see equation 2 below). 
Equation 2: $y_{t+1}=f\left(\theta_{t}\right)$

The present paper aims to investigate the dynamic relations between emotion, appraisal and cognitive achievement, by using a method that can assess the non-linear relation between the components, which are involved in a net of reciprocal causality. The paper follows the dynamic approach tradition also employed in the personality (Cramer et al., 2012a; Schmittmann et al., 2013) and developmental literature (Van Geert \& Fischer, 2009; Van Geert \& Steenbeek, 2005b; Yan \& Fischer, 2002).

\section{Methodology of Research}

\section{Sample of Research}

Thirteen students, seven male and six female, responded all items of the instruments used in the research. Four of them were in seventh grade, three in eighth grade, and six in the ninth grade. Mean age was 13.53 years and standard deviation was 1.05. All students were from a private school located in the city of Belo Horizonte, Minas Gerais, Brasil.

\section{Instrument and Procedures}

\section{Inductive Reasoning Developmental Test (IRDT)}

The Inductive Reasoning Developmental Test (IRTD) is a pencil-and-paper instrument designed to assess developmentally sequenced and hierarchically organized inductive reasoning. The sequence was constructed based on the Model of Hierarchical Complexity, and has eight items specifically designed to identify each stage, covering seven stages (from Pre-operational to Metasystematic) in a total of 56 items. Each item is composed of four letters, or sequence of letters, with a specific rule (correct items), plus one letter or sequence with a different rule (exception). The task is to discover which letter or sequence is the exception. Golino and Gomes (2012) administered the IRDT to 1,193 Brazilian people ( $53 \%$ women, $47 \%$ men) from 6 to 86 years $(\mathrm{M}=15.02, \mathrm{SD}=$ 12.30). The dichotomous Rasch Model was used, and pointed a separation reliability of 0.99 for the 56 items and an infit mean square mean of $0.90(\mathrm{SD}=0.23)$. The person separation reliability was 0.80 . The principal contrast showed that the raw variance explained by measures was $73.2 \%$, and that the unexplained variance in the first contrast was $6.9 \%$, suggesting the unidimensionality of the instrument. The items' difficulties presented 7 clusters with gaps between them, as visually verified by the Wright map. The clusters were confirmed by the hidden markov model, which presented a solution with 7 classes $(\log$ Lik. $=-26.28$, df=62, AIC: 176.57 , BIC: 302.14$)$. In the current study only the first five difficulty levels were employed. The mean difficulty (Rasch score) of each level, its standard deviation, as well as the minimum and maximum difficulty for each level are presented in table 1 . In the current study, only five levels of difficulty were employed.

From the items of the Inductive Reasoning Developmental Test four booklet versions were created. Each booklet version has 60 items, forming 4 cycles. Each cycle has five groups of three items, each one representing a specific level of difficulty. So, the first 15 items of the booklet composes the first cycle: items one, two and three represents the first difficulty level; items four, five, and six represents the second difficulty level, and so on, until items 13,14 and 15 that represents the fifth difficulty level. The next 45 items compose the second, third and fourth cycle, organized with the same structure as cycle one. So, each booklet version is composed by four cycles (see Figure 1). Each cycle has one item that is also present in another cycle of the same booklet. Repeating the same item twice along the booklets is a strategy that allows equating using Item Response Theory techniques, and may be useful in future studies employing the data available in the current paper. The difference between the booklet versions is the order of the four cycles, e.g., the first cycle of the version one is the fourth cycle of the version four. Table 1 shows one example of item from each of the five difficulty levels that composes a cycle, as well as the mean difficulty, standard deviation, 
minimum and maximum difficulty per level (using logits as unit of measurement). Although table 1 shows the mean difficulty of each level using the Rasch difficulty of the items, in the current study we are using the item's raw score. The raw score is generated through the sum of right answers in each group of three items representing the difficulty levels in each cycle. Each group has a score that goes from 0 (participant failed all three items) to 3 (participant passed all three items).

Table 1. Example of items of each difficulty level, mean, standard deviation, minimum and maximum difficulty (logits) for each level.

Level three

\section{Appraisal of Test Achievement and Emotional Valence}

Along with each group of three items (representing the difficulty levels) there is an achievement appraisal scale. The participants answer how many items they believe to have passed, from three to zero items of that group. They are asked to do it for each group of three items. The score for each difficulty group goes from 0 (they believe to have failed all three items) to 3 (they believe to have passed all three items). Together with the appraisal scale, the participants mark one out of five emotions that represent different emotional valences: from a very negative (score 1) to a very positive (score 5).

\section{Data Analysis}

The data were collected in March of 2011. Each participant answered only one booklet version in a unique moment. Different participants responded different booklet versions. After the ending of one cycle of the booklet, it was given one minute of pause before the beginning of the next cycle.

The answers of each participant generate 20 raw scores of test achievement: Each group of three items produces a raw score of zero, one, two or three points (respondent passed all three items). Twenty raw scores of emotional valence and achievement appraisal were also produced. 
One polychoric matrix was generated by person, aiming the investigation of the profiles of correlation between test achievement, appraisal and emotional valence. After estimating the polychoric correlation, a Hidden Markov Model (Baum \& Petrie, 1966) was applied to investigate the states involved in the dynamic of the test achievement, appraisal, and emotional valence. In order to apply the Hidden Markov Model, the depmixS4 package (Visser \& Speekenbrink, 2010) of the $\mathrm{R}$ software was used. Hidden Markov Model (HMM) is a probabilistic model that specifies a finite set of hidden (or latent) states (or classes with transition probabilities) that causes an observable outcome. The HMM is based on two assumptions: 1) the current state depends only on the previous state (first-order Markov Process), and 2) observable outcomes are dependent only on the current state, at time t. Figure 2 below illustrates an HMM:

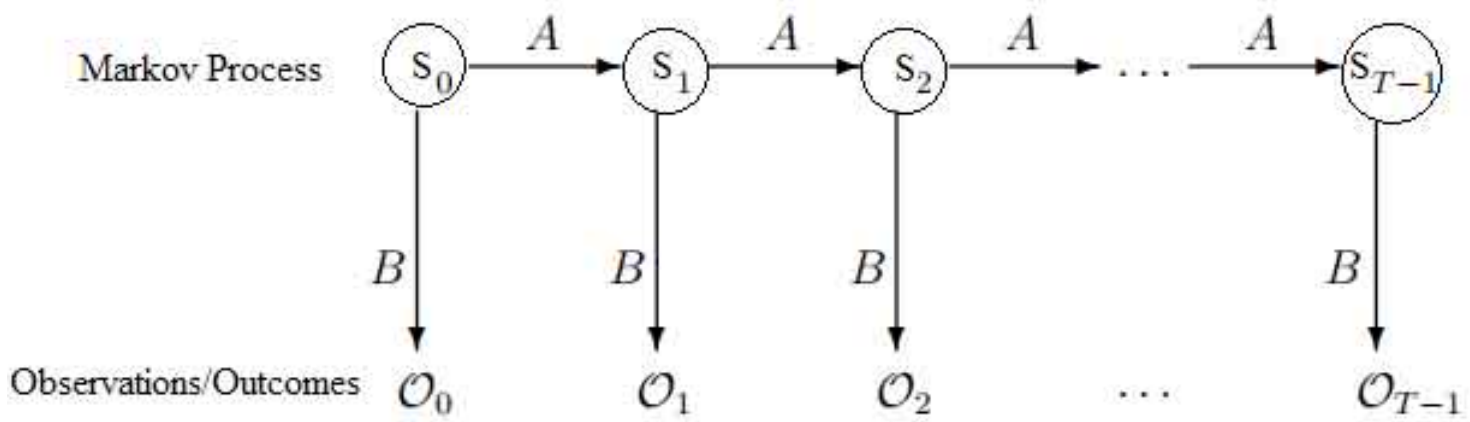

Figure 2: Markov Process.

Where $S_{n}$ represents states sequence from 0 to T-1, $A$ represents state transition probabilities, $B$ observation/outcome matrix probabilities and $\mathrm{O}_{\mathrm{n}}$ observation/outcome sequence from 0 to $\mathrm{T}-1$. Transition probabilities between states are assumed to follow first-order Markov process, i.e. state at current time depend on the previous state (Visser \& Speekenbrink, 2010). Every state has a probability of remaining unchangeable, and a probability of transiting for any other state (see figure 3 ).

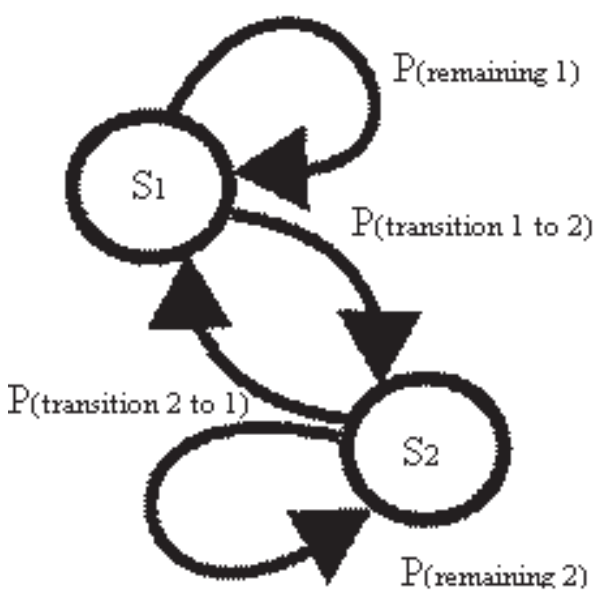

Figure 3: Transition between states.

This particular characteristic of the HMM is interesting to our investigation since the first hypothesis states that different patterns of correlation between cognitive achievement, appraisal and emotions will be found in our sample. So, the use of HMM allows the identification of each state that emerges from the interactions between achievement, appraisal and emotions. For example, state $\mathrm{S}_{1}$ may represent a state in which the person presents high probability of getting a score of zero in the cognitive test, a high probability of judging the cognitive achievement as low and a high probability of experiencing very negative emotions. Continuing the example, state $\mathrm{S}_{1}$ may represent a 
state in which the person presents high probability of getting a score of three in the cognitive test, a high probability of judging the cognitive achievement as high and a high probability of experiencVolume 6, 2013 ing positive very emotions. Furthermore, the HMM allows the calculation of the probabilities of changing from state $\mathrm{S}_{1}$ to state $\mathrm{S}_{2}$.

In sum, an HMM has two main parts: states' measurement model and transition between states. States are the construct under investigation (in our case it is the relation between test achievement, appraisal and emotion), and transition characterizes the dynamics between states over time (Visser, Raijmakers \& Van der Maas, 2009). A latent variable explains the dependencies between each state of dynamic system and its previous state. Eight models were estimated, from 1 to 8 states. Each state represents a different relation between test achievement, appraisal and emotional valence. The existence of more than one state indicates distinct relations between the components of the system. In order to choose the best model, two indexes were employed: Akaike's Information Criterion (AIC; Akaike, 1973) and Bayesian Information Criterion (BIC; Schwarz, 1978).

Since the depmixS4 package uses the expectation-maximization (EM) algorithm to maximize the log-likelihood, the AIC and BIC values can range due to random initialization of this algorithm. In this case, several interactions are necessary to estimate the global minimum of the AIC and BIC, instead of the local minimum (Haughton, Legrand, \& Woolford, 2009). So, two hundred models were calculated for the estimation of the AIC and BIC for each number of states, from 1 to 8 .

\section{Results of Research and Discussion}

We will present in the same section results and discussion due to the necessity to describe the results along with the interpretation of the qualitative profiles and the states identified through the Hidden Markov Model. Seven qualitative profiles were identified, indicating different patterns of correlations between the variables of the study. These profiles probably imply different functional aspects of the system. Next, we describe each profile, presented in the Table 2.

Table 2. The Seven Qualitative Profiles: correlation matrixes and graphics.

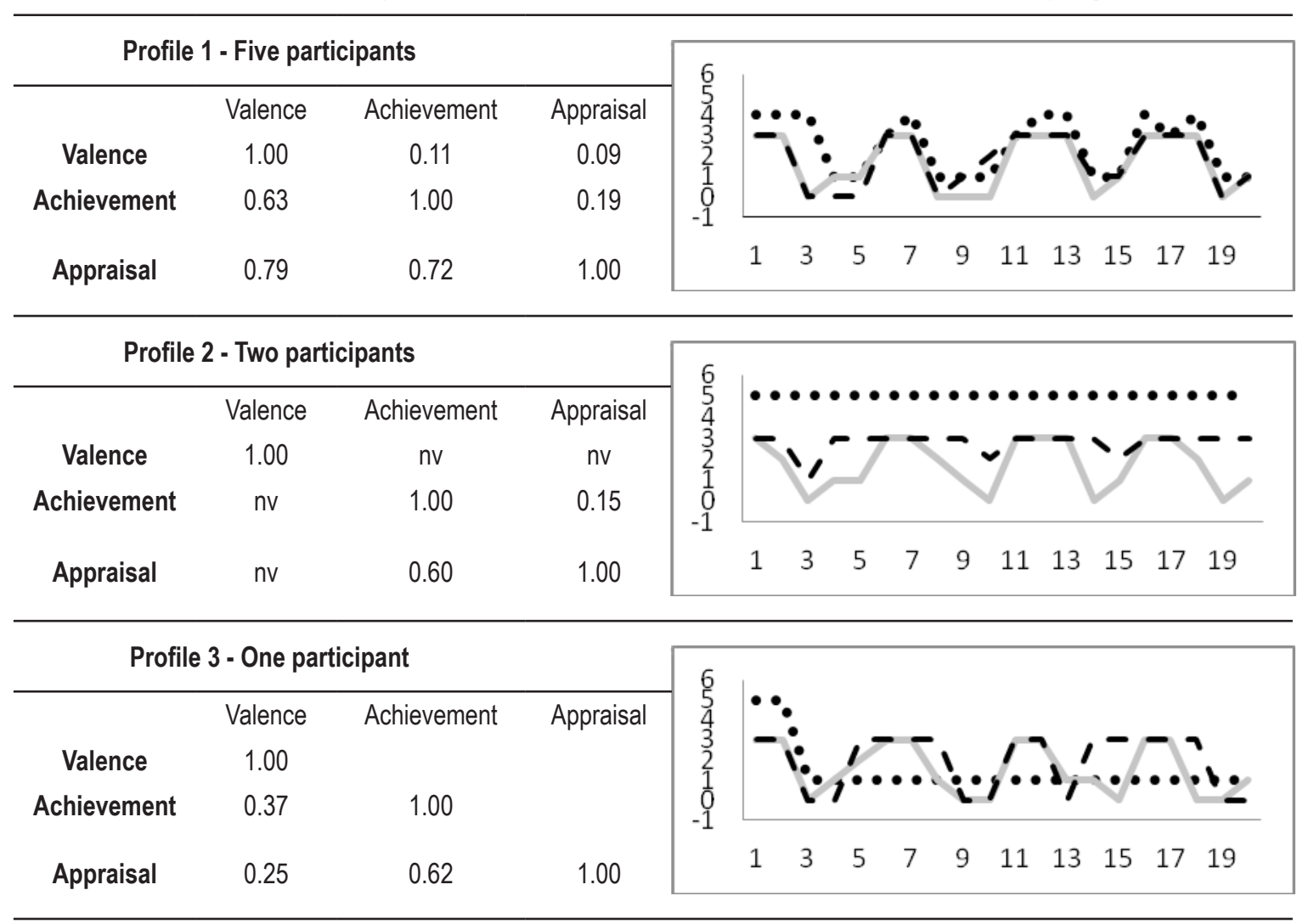




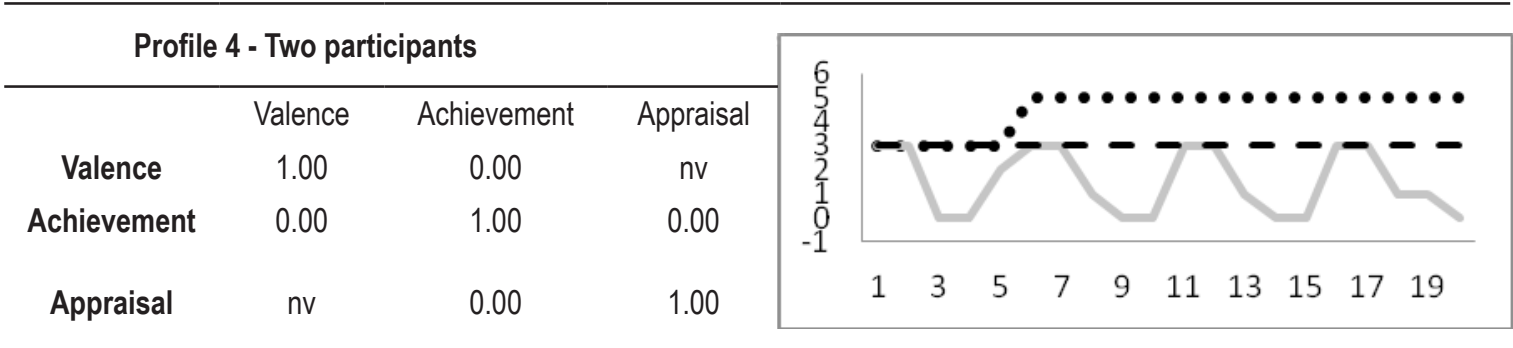

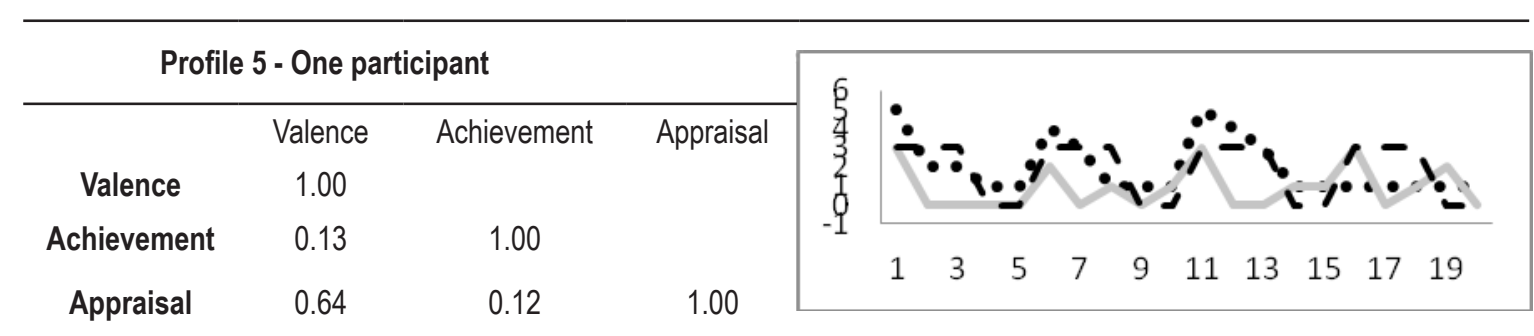

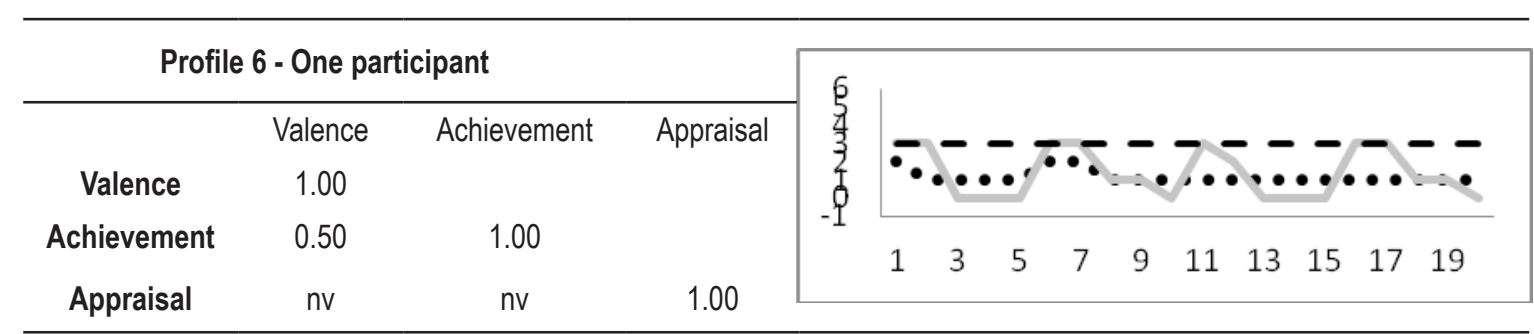

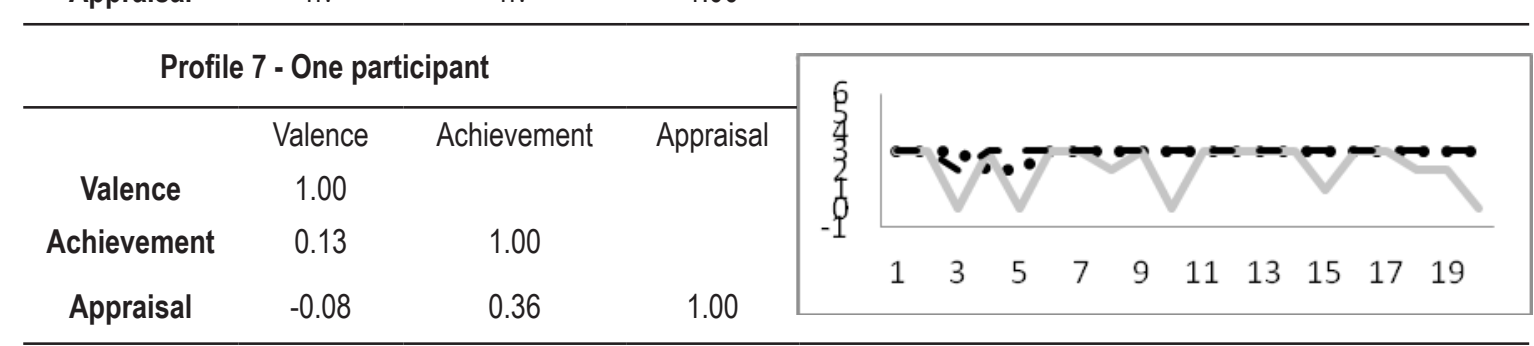

$n v=$ No variation; Dotted line = Emotional Valence; Dashed line = Appraisal; Grey line = Cognitive Achievement

The correlation matrix of the profiles, with more than one participant each, shows the mean values below the diagonal and standard deviation upper the diagonal

In profile one, which was found in five participants, there were relatively moderate correlations between all the variables, indicating that the appraisal was reasonably connected to emotional valence $(0.79)$ and at least reasonably linked to test achievement (0.72). Achievement and emotional valence were also moderately related (0.63). People with this profile are relatively good at assessing their performance, and the emotional valence has a function of evaluation somehow similar to the appraisal ability. If their performance becomes poor in the difficult items they will perceive this situation and judge their achievement as a poor one. At the same time they will feel a certain discomfort and displeasure. When the items become easier and the person passes them all, he/she will perceive this situation and judge to have passed all items. They will also report more comfort and pleasure compared with the previous situation. In profile one the appraisal can be thought as a "cold" evaluative ability, while the emotional valence can be thought as a "hot" evaluative ability of the performance, making an analogy with the cold and hot executive functions of neuropsychology (Metcalfe \& Mischel, 1999).

Profile two had two participants. In this profile, there were a moderate correlation $(0.60)$ between appraisal and test achievement and an absence of variation in the emotional valence, indicating that these participants only show a "cold" evaluative function of the performance. The "hot" evaluative function did not appear in this profile. Table 2 shows a graphic of one person that presents only the maximum positive emotional valence, independently of his/her achievement or his/her appraisal. It is possible that emotion, in this case, works as a protective strategy, since a strong and rigid emotional valence would protect against bad feelings and displeasures in the moments of low achievement. But it is also possible that this characteristic would reflect a dysfunctional way of reacting to difficulty. Both possibilities need to be investigated in future studies. 
Profile three, found in one participant, presented moderate correlation between achievement and appraisal (0.62), but showed weak correlations between emotional valence and appraisal (0.25), Volume 6, 2013 and between emotional valence and test achievement $(0.37)$. These characteristics indicate a person that is moderately good in judging his/her achievement. However, the "cold" and "hot" evaluative functions of the performance have a weak relationship, possibly indicating a low communication between these functions. This low communication would be thought as a gap between the rational ("cold") and the intuitive ("hot") evaluation, which would be dysfunctional. In Table 2, the person with the third profile showed a very positive emotional valence when at the beginning of the test. However when he/she started to fail the items, he/she strongly dropped out the emotional valence to a very negative one, and remained in this condition throughout the whole test. In this case, the impact of the first failed items probably was potentially damaging and the emotional valence lost, at least in part, the capability of reflecting the test achievement. The previous interpretative comments are speculative and need to be investigated in future studies.

Profile four was found in two participants and can be thought as dysfunctional because there was no correlation between the three variables. Appraisal was completely unattached to test achievement and to emotional valence. There was a disintegration or non-integration of the system, indicating an independency of the components.

Profile five was found in one participant and is similar to profile one because both had a moderate correlation between appraisal and emotional valence, indicating that the "cold" and "hot" function are connected. However, both appraisal and emotional valence showed a very weak correlation with test achievement ( 0.12 and 0.13 , respectively), indicating that a participant with profile five is a bad judge. The major difference between profile one and profile five seems to be that the first is a reasonably good judge while the second is a bad judge.

Profile six was found in one participant and had a dysfunctional characteristic because the appraisal does not vary in function of the achievement. However, the emotional valence had a moderate correlation with the test achievement, indicating that the "hot" component composes the evaluative function of the performance, while the "cold" component showed a disarticulation with the other two components of the system.

Finally, profile seven, found in one participant, had a weak correlation between appraisal and test achievement and a very weak correlation between emotional valence and appraisal, as well as between emotional valence and test achievement. This condition is relatively similar to profile four. It also indicates a gap between the "cold" and "hot" evaluative functions.

After the presentation of the qualitative profiles, the four states found using the Hidden Markov Model will be presented. Eight models were compared. The model number one represents the presence of only one state of the system, while model two represents the presence of two states, and so on. Table 3 presents the logs of the models, their degree of freedom, as well as the AIC and BIC values. The BIC criterion points the fourth model as the best one $(\mathrm{BIC}=1675.66)$, while the AIC criterion points the sixth model as the one presenting a better data fit $(\mathrm{AIC}=1427.48)$. Considering that BIC is more parsimonious than AIC, the fourth model was chosen.

Table 3. The Seven Qualitative Profiles: correlation matrixes and graphics.

\begin{tabular}{ccccc}
\hline Models & $\log$ & df & AIC & BIC \\
\hline 1 & -912.02 & 10 & 1844.03 & 1879.64 \\
2 & -817.01 & 23 & 1680.03 & 1761.93 \\
3 & -738.57 & 38 & 1553.14 & 1688.44 \\
4 & -684.91 & 55 & 1479.83 & 1675.66 \\
5 & -642.42 & 74 & 1432.84 & 1695.33 \\
6 & -618.74 & 95 & 1427.48 & 1765.75 \\
7 & -598.84 & 118 & 1433.69 & 1853.85 \\
8 & -581.10 & 143 & 1448.19 & 1957.37 \\
\hline
\end{tabular}

$\log =$ Loglikelihood; $d f=$ Degree of Freedom; AIC = Akaike's Information Criterion; BIC = Bayesian Information Criterion 
The four states model represents qualitative processes found by a quantitative method (Hidden Markov Model). The measurement model for each of the states shows distinct score probabilities for each component of the system (see table 4).

A person in the state one has a probability of $45 \%$ to declare a very negative emotional valence (score 1 ) and $35 \%$ to declare a neutral emotional valence (score 3 ). There is $16 \%$ of probability to inform a negative emotional valence (score 2), a 4\% of probability to report a positive emotional valence (score 4), and a very low probability (approximately zero) of score 5 (very positive valence). However, the $16 \%, 4 \%$ and almost $0 \%$ are below the chance probability in the scale of five points, where the participant have $20 \%$ of chance to randomly choose any of the five options. In this case, only the very negative emotional valence and the neutral emotional valence reached a value greater than chance and will be considered in state's one interpretation. State one shows a considerable probability of someone to report neutral or negative feelings. There is a major probability that the test achievement of a person in this state would be low, because there is $67 \%$ of probability to fail all the three items of an item group, and $25 \%$ of probability to pass only one item. A person in this state probably judges to have failed all three items (probability $=63 \%$; the only value that is greater than chance). In sum, state one represents a moment in the dynamic system that a participant probably fails all items, or passes only one in the group of three items, appraisals to have failed all items, and experiences neutral valence or bad feelings and displeasure. There is in state one a good connection between failing the items, judging as a poor performance and feeling bad about it.

Table 4. The Four States and the Probability of the Score Categories in Test Achievement, Appraisal, and Emotional Valence.

\begin{tabular}{|c|c|c|c|c|c|c|c|}
\hline State & $\begin{array}{c}\text { State's } \\
\text { component }\end{array}$ & & & Scores & & & Sum \\
\hline & & 1 & 2 & 3 & 4 & 5 & \\
\hline \multirow{3}{*}{ State 1} & Valence & 0.45 & 0.16 & 0.35 & 0.04 & 0.00 & 1.00 \\
\hline & Achievement & 0.67 & 0.25 & 0.08 & 0.00 & & 1.00 \\
\hline & Appraisal & 0.63 & 0.14 & 0.16 & 0.07 & & 1.00 \\
\hline \multirow{3}{*}{ State 2} & Valence & 0.00 & 0.00 & 0.00 & 0.00 & 1.00 & 1.00 \\
\hline & Achievement & 0.23 & 0.19 & 0.06 & 0.52 & & 1.00 \\
\hline & Appraisal & 0.00 & 0.02 & 0.04 & 0.94 & & 1.00 \\
\hline \multirow{3}{*}{ State 3} & Valence & 0.00 & 0.00 & 0.69 & 0.31 & 0.00 & 1.00 \\
\hline & Achievement & 0.18 & 0.05 & 0.12 & 0.66 & & 1.00 \\
\hline & Appraisal & 0.00 & 0.00 & 0.04 & 0.96 & & 1.00 \\
\hline \multirow{3}{*}{ State 4} & Valence & 0.84 & 0.14 & 0.00 & 0.00 & 0.02 & 1.00 \\
\hline & Achievement & 0.41 & 0.21 & 0.06 & 0.33 & & 1.00 \\
\hline & Appraisal & 0.21 & 0.00 & 0.00 & 0.79 & & 1.00 \\
\hline
\end{tabular}

Score meaning:

Valence: 1 - Very negative emotional reaction; 2 - Negative emotional reaction; 3 - Neutral emotional reaction; 4 - Positive emotional reaction; 5 - Very positive emotional reaction.

Achievement: 1 - No right answers; 2 - One right answer; 3 - Two right answers; 4 - Three right answers.

Appraisal: 1 - Participant reports his/her achievement on the test as having no right answers; 2 - Participant reports his/her achievement on the test as having only one right answer; 3 - Participant reports his/her achievement on the test as having two right answers; 4 - Participant reports his/her achievement on the test as having three right answers.

State three represents the opposite of state one, so it will be described before state two. The person who's in state three, shows a neutral or a positive emotional valence ( $69 \%$ and $31 \%$, respectively), tends to passes all three items of a group (66\%) and tends to strongly judge to have passed all items (96\%). Concluding, state three represents a tendency to pass all items, appraising to have passed all items and feeling neutral or positive emotions. Concluding, state three represents a tendency to pass 
all items, appraising to have passed all items and feeling neutral or positive emotions. In this state there is an overestimation of the cold evaluation, the person has $96 \%$ of chance to evaluate having passed all items whereas the chance of actually having passed them is of $66 \%$.

State two is interesting because there is almost $100 \%$ of probability that someone in this state feels a strong positive emotional valence. There is a powerful pleasure and positive feelings involved in this case. The only probability greater than chance in test achievement is the person passes all items $(52 \%)$. Also, the person in state two has a very high likelihood of judging to have passed all items (94\%). State two represents a rigid and very positive emotional reaction, with a pattern of high achievement (52\%) and an appraisal that overestimates this achievement pattern.

State four is almost the opposite of the state two, but not entirely. There is a very negative emotional valence $(84 \%)$ in this state, which is the opposite of state two. However, the test achievement is different. People in this state can fail all items (41\%) or pass all items $(33 \%)$ of a group of three items. In this state the relatively similar probability in given all wrong or all right answers to the items is connected to a very negative feeling. Finally, people in this state tend to judge that they passed all items $(79 \%)$ much more than the probability of really passing them $(33 \%)$, indicating a relatively bad precision in the appraisal. Concluding, state four represents a very negative emotional valence, a similar probability to fail or to pass all items and a tendency to overestimate the performance.

Concerning the quality of the appraisal, in state one a good judgment is shown, since the appraisal of achievement is similar to the observed achievement. In state two and three a moderate judgment is present, since the pattern of achievement is high but there's a considerable part of chance (48\%) to give none, one or two right answers that is underestimated. State four shows a poor judgment, because there is a bimodal chance of failing all items or passing all items and the appraisal overestimates the probability of high achievement and underestimates the probability of low achievement.

The results also show state's transition probabilities (see table 5). State one is the less stable, presenting $54 \%$ of probability of not changing into any other state. However, there is a probability of $13 \%$ of changing into state two and a probability of $27 \%$ of changing into state three. State one also has a probability of $6 \%$ of changing to the forth state. State two has a strong probability to stay the same $(83 \%)$. There is a probability of $11 \%$ of changing into state one, a probability of $6 \%$ of changing into state three, with almost $0 \%$ chance of changing into state four. State three also has a strong probability to stay the same $(80 \%)$, with a probability of $19 \%$ of changing into state one, a chance of $1 \%$ of changing to state two, and almost $0 \%$ chance of changing into state four. State four is the most stable, presenting $94 \%$ probability of staying the same, with a small probability of changing into state three $(6 \%)$, with almost $0 \%$ chance of changing both to state one or state two.

Table 5. Transition probabilities between the four states.

\begin{tabular}{ccccc}
\hline & State 1 & State 2 & State 3 & State 4 \\
\hline State 1 & 0.54 & 0.11 & 0.19 & 0.00 \\
State 2 & 0.13 & 0.83 & 0.01 & 0.00 \\
State 3 & 0.27 & 0.06 & 0.80 & 0.06 \\
State 4 & 0.06 & 0.00 & 0.00 & 0.94 \\
Sum & 1.00 & 1.00 & 1.00 & 1.00 \\
\hline
\end{tabular}

In sum, state one is relatively fluid because it has a $46 \%$ chance of changing to another state. It is more likely that state one (low achievement, neutral or negative emotions and a tendency to correctly judge the performance) transforms into state three (high achievement, neutral or positive emotions and a tendency to moderately judge the performance), then to any other state. This tendency is reciprocal, i.e. it is more likely that state three transforms into state one than to any other state. State two, state three and state four are more inflexible since they have a probability of, at least, $80 \%$ of staying the same. State four is the most rigid. If changing, state two is more likely to transform into state one, while state four is more likely to transform into state three. State one is the only one that has at least $5 \%$ of probability to transform into all the other states. 


\section{Conclusions}

The present study applies the dynamic system approach to investigate the reciprocal relation between cognitive performance, achievement appraisal and self-reported emotional reactions. Employing a specific test design, with four cycles of items with different levels of increasing difficulty, we presented two hypotheses: 1) More than one correlation pattern between test achievement, appraisal and emotion will be found and 2) test achievement, appraisal and emotional valence form a dynamic system that is explained by a latent variable that depends on the previous state of the system (see figure 2). This latent variable explains the dependency relationship between the observable variables (i.e. between achievement, appraisal and emotions). So, an observable relation $\left(O_{2}\right)$ between achievement, appraisal and emotions of time $T_{2}$ are explained by the state $S_{2}$. By its turn, $S_{2}$ is dependent on the previous state $S_{l}$ of time $T_{l}$. State $S_{l}$ also explains the observable relation $O_{l}$. The results showed seven qualitative distinct patterns of correlation between the three variables under investigation as well as a four-state Hidden Markov Model. Both the correlation profiles and the four states model was presented and discussed in the previous section. However, some highlights will be made in the next paragraphs.

We discover that there is a good probability that each state remains unchangeable. However, improving the probability of a high cognitive performance and the probability of judging this performance as good (giving right answers to the three items) may lead to positive emotional reactions, as verified by the probability of changing from state 1 or 4 to state 3 . On the other hand, decreasing the probability of a high cognitive performance and the probability of judging this performance as good may lead to negative emotional reactions, as verified by the probability of changing from state 2 or 3 to state 1 . Both examples, whose interpretation focuses on the idea that improving cognitive performance may leads to positive emotional reaction, and worsening cognitive performance may leads to negative emotional reaction, is just one way to address the matter. The other ways are represented by three ideas.

First, that increasing the probability of experiencing positive emotions may increase the probability of having a high cognitive performance and the probability of judging this performance as a good one (going from state 1 to state 2 or 3 ). Second, that increasing the probability of experiencing negative emotions may decrease the chance of a high cognitive performance, improve the chance of a poor performance and diminish the probability of judging the performance as good (going from state 3 to 4). And third, that decreasing the probability of experiencing positive emotions and increasing the chance of expressing negative emotions may increase the probability of a low cognitive performance and the chance of judging this performance as a poor one.

A question someone may ask is: which interpretation is correct: 1) changes in cognitive performance impact emotional reactions, or 2) changes in the emotional reactions impact cognitive performance? The answer is: probably both are right! Cognitive performance and emotions walk in the same direction when there is a moderately good judgment of the performance, i.e. people tend to give three correct answers and to judge their answers as correct or fail the items and judge their answers to be wrong. Both statements are true: cognitive performance influence emotional reactions and emotional reactions influence cognitive performance. Achievement appraisal, cognitive performance and emotional reactions form a complex dynamic system. They influence each other in a bidirectional way, creating a feedback loop that shapes their relation. This bidirectional influence is also reported in the personality literature (Cramer et al., 2012a; Cramer et al., 2012b).

The relation between emotions and intellectual achievement has been described before, but without employing a dynamical system approach (Pekrun, 2006; Pekrun \& Sthepens, 2010). If cognitive performance, emotions and appraisal are studied using any model that complies with the basic equation 1, presented in the introduction, will be possible to discover the net of reciprocal causality between them. And that is what we did in the current study. The application of the dynamical system approached allowed discovering that different profiles of correlation between cognitive performance, emotions and appraisal form four different states. Furthermore, we discovered that these states are stable, but when analyzing their transition probabilities, we verified a pattern indicating that changing the cognitive performance may leads to changes in emotional reaction and that changing 
emotional reactions may leads to changes in cognitive performance, provided that performance is well appraised.

Before the application of dynamic models in the current study, or in the above mentioned personality discussions (Cramer et al., 2012a; Cramer et al., 2012b), van Geert and Fischer (2009), Van Geert and Steenbeek (2005b) and Yan and Fischer (2002) applied it in studies of developmental psychology. This approach brings two main contributions to psychology: 1) it defines a complex system as a system in which the components interacts in a non-linear and self-organized way to define the owner structure; and 2) it allows diminishing the complexity without losing the fundamental properties of the system. Both contributions are illustrated in the present paper, with the results of the dynamic system formed by cognitive performance, achievement appraisal and emotions. Future studies should employ a larger sample in order to create more stable correlation matrixes between the variables under investigation.

\section{Acknowledgements}

The current research was supported by the Fundação de Amparo à Pesquisa de Minas Gerais - FAPEMIG (Foundation for Research Support from the State of Minas Gerais, Brasil).

\section{References}

Akaike, H. (1973). Information theory and an extension of the maximum likelihood principle. In B. N. Petrov \& F. Csaki (Eds.). Second international symposium on information theory (pp. 267-281). Budapest: Academiai Kiado.

Baum, L. E. \& Petrie, T. (1966). Statistical Inference for Probabilistic Functions of Finite State Markov Chains. The Annals of Mathematical Statistics, 37 (6), 1554-1563.

Cramer, A. O. J., Van der Sluis, S., Noordhof, A., Wicherts, M., Geschwind, N., Aggen, S. H., Kendler, K. S., \& Borsboom, D. (2012a). Dimensions of normal personality as networks in search of equilibrium: You can't like parties if you don't like people. European Journal of Personality, 26 (4), 414-431.

Cramer, A. O. J., Van der Sluis, S., Noordhof, A., Wicherts, M., Geschwind, N., Aggen, S. H., Kendler, K. S., \& Borsboom, D. (2012b). Measurable like temperature or mereological like flocking? On the nature of personality traits. European Journal of Personality, 26 (4), 451-459.

Golino, H. F. \& Gomes, C. M. A. (2012). The structural validity of the Inductive Reasoning Developmental Test for the Measurement of Developmental Stages. In Adult Development: Past, Present and New Agendas of Research. Symposium conducted at the meeting of the European Society for Research on Adult Development, Coimbra, PT. Retrieved from http:/www.europeadultdevelopment.org/materials1.html

Haughton, D. M., Legrand, P., \& Woolford, S. W. (2009). Review of Three Latent Class Cluster Analysis Packages: Latent Gold, poLCA, and MCLUST. The American Statistician, 63 (1), 81-91.

Metcalfe, J., \& Mischel, W. (1999). A two system analysis of delay of gratification. Psychological Review, $106(1), 3-19$.

Mitra, S., Tooley, J., Inamdar, P., \& Dixon, P. (2003). Improving English Pronunciation: An Automated Instructional Approach. Information Technologies and International Development, 1 (1), 75-84.

Pekrun, R., \& Stephens, E. J. (2010). Achievement Emotions. A control-value approach. Social and Personality Psychology Compass, 4 (4), 238-255.

Pekrun, R. (1992). The impact of emotions on learning and achievement: Towards a theory of cognitive/motivational mediators. Applied Psychology: An International Review, 41 (4), 359-376.

Pekrun, R. (2006). The control-value theory of achievement emotions: assumptions, corollaries, and implications for educational research and practice. Educational Psychology Review, 18 (4), 315-341.

Schmittmann, V. D., Cramer, A. O. J., Waldorp, L. J., Epskamp, S., Kievit, R. A., \& Borsboom, D. (2013). Deconstructing the construct: A network perspective on psychological phenomena. New Ideas in Psychology, 31 (1), 43-53.

Schwarz, G. (1978). Estimating the Dimension of a Model. Annals of Statistics, 6 (2), 461-464.

Van Geert, P., \& Steenbeek, H. (2005a). Explaining after by before. Basic aspects of a dynamic systems approach to the study of development. Developmental Review, 25 (34), 408-442. 
ISSN 2029-8587

PROBLEMS

OF PSYCHOLOGY

IN THE $21^{\text {st }}$ CENTURY Volume 6, 2013

28

Van Geert, P., \& Steenbeek, H. (2005b). The dynamics of scaffolding. New Ideas in Psychology, 23 (3), $115-$ 128.

Van Geert, P., \& Fischer, K. W. (2009). Dynamic Systems and the Quest for Individual-Based Models of Change and Development. In J. P. Spencer, M. S. C. Thomas, \& J. L. McClelland (Eds.), Toward a Unified Theory of Development Connectionism and Dynamic System Theory Re-Consider (pp. 313-336). Oxford: Oxford University Press.

Van Geert, P., \& Steenbeek, H. (2008). A complexity and dynamic systems approach to developmental assessment, modeling and research. In A. M. Battro, K. W. Fischer \& P. Léna (Eds.). The educated brain: essays in neuro-education (pp. 71-94). Cambridge U.K.: Cambridge University Press.

Visser, I., \& Speekenbrink, M. (2010). depmixS4: An R Package for Hidden Markov Models. Journal of Statistical Software, 36 (7), 1-21. URL http://www.jstatsoft.org/v36/i07/.

Visser, I., Raijmakers, M. E. J., \& Van der Maas, H. L. J. (2009). Hidden Markov models for individual time series. In J. Valsiner, P. C. M. Molenaar, M. C. D. P. Lyra, \& N. Chaudhary (Eds.). Dynamic Process Methodology in the Social and Developmental Sciences (pp. 269-289). Springer, New York.

Yan, Z. \& Fischer, K. (2002). Dynamic variations in adult cognitive microdevelopment. Human Development, 45 (3), 141-160.

Advised by Stanislava Yordanova Stoyanova, South-West University "Neofit Rilski", Bulgaria

Received: June 04, 2013

Accepted: July 06, 2013

Cristiano M. A. Gomes Ph.D, Adjunct Professor, Federal University of Minas Gerais, School of Philosophy and Human Sciences, Av. Antônio Carlos, 6627, Room 4010, Belo Horizonte, Minas Gerais, CEP. 31270-901, Brazil.

E-mail: cristianogomes@ufmg.br

Website: http://laicoufmg.wix.com/laico

Hudson F. Golino $\quad$ MSc., B.Psy, Ph.D Student of Neurosciences, Assistant Researcher, Federal University of Minas Gerais, School of Philosophy and Human Sciences, Av. Antônio Carlos, 6627, Room 4010, Belo Horizonte, Minas Gerais, CEP. 31270-901, Brazil.

E-mail: hfgolinoh@gmail.com

Website: http://laicoufmg.wix.com/laico

Bianca C.G. Costa

B.Psy., Master Student of Developmental Psychology, Assistant Researcher, Federal University of Minas Gerais, School of Philosophy and Human Sciences, Av. Antônio Carlos, 6627, Room 4010, Belo Horizonte, Minas Gerais, CEP. 31270-901, Brazil. E-mail: biacgc@gmail.com Website: http://laicoufmg.wix.com/laico 